\title{
The effects of meningitis on anterior pituitary functions
}

\author{
Suat Zengin, Behcet Al, Mehmet Dogan, Basri Can, Mustafa Bogan, Cuma Yildirim
}

Gaziantep University School of Medicine, Department of Emergency Medicine Gaziantep, Turkey

\section{Email address:}

zengins76@gmail.com(S. Zengin)

\section{To cite this article:}

Suat Zengin, Behcet Al, Mehmet Dogan, Basri Can, Mustafa Bogan, Cuma Yildirim. The Effects of Meningitis on Anterior Pituitary Functions. American Journal of Health Research. Vol. 1, No. 3, 2013, pp. 36-41. doi: 10.11648/j.ajhr.20130103.11

\begin{abstract}
Objective: This study investigated the changes in the anterior pituitary hormones of patients who were admitted to an emergency service and diagnosed with acute meningitis. Methods: A total of 21 patients who were admitted to an emergency service between 01 January and 31 October 2012, and diagnosed with meningitis, were included. Blood samples were collected from the patients within an initial 24 hours of admission and at month 6 , in order to measure thyroid-stimulating hormone (TSH), growth hormone $(\mathrm{GH})$, follicle-stimulating hormone (FSH), luteinizing hormone $(\mathrm{LH})$, prolactin (PRL), and adrenocorticotropic hormone (ACTH) levels. The results were analyzed using SSPS-18 software. Results: Of the patients, $47.6 \%$ were male, and $52.4 \%$ were female. At month 6 , TSH, LH, and ACTH levels were higher than those measured on admission. This difference was meaningful for TSH, but insignificant for LH and ACTH. At month 6, mean GH, FSH, and PRL levels were lower than admission levels. At the time of admission, FSH was at lower than normal levels in seven patients, LH was lower in six patients, TSH was lower in four patients, GH was lower in four patients, and ACTH in was lower in three patients. At month 6, FSH was lower in five patients, LH was lower in four patients, TSH was lower in one patient, GH was lower in six patients, and ACTH was lower in five patients. Conclusion: The differences between hormone levels at admission and at month 6 show that meningitis causes changes in anterior pituitary hormones.
\end{abstract}

Keywords: Meningitis, Anterior Pituitary Function, Changes in Anterior Pituitary Hormones

\section{Introduction}

In addition to many other functions, pituitary hormones are also responsible for appropriate responses to stress and the sustaining of vital bodily functions. Hypopituitarism (failure of the pituitary gland) is a clinical syndrome that develops as a result of insufficient production and release of one or more of the pituitary hormones. Pituitary insufficiency may have diverse causes, the most common being pituitary adenoma, craniopharyngioma, Sheehan's syndrome, lymphocytic hypophysitis, infiltrative diseases, irradiation, and traumatic brain injury or surgery [1,2].

Meningitis is an inflammatory disease of the pia mater, arachnoid and cerebrospinal fluid (CSF) that is caused by bacterial or viral pathogens. Tuberculous meningitis, which is a common cause of bacterial meningitis, has long been known to cause pituitary dysfunction (3). Among the various causes of pituitary insufficiency, infectious diseases of the central nervous system (CNS) are relatively rare, and have primarily been reported in only three retrospective and two prospective studies [1,4-7].
Here, we present the results of a prospective study investigating pituitary function in patients with acute infectious meningitis, both in the acute phase and 6 months after the initial event.

\section{Patients and Methods}

A total of 21 patients who were admitted to the Emergency Service of the Faculty of Medicine, Gaziantep University, between 01 January and 31 October 2012, and diagnosed with meningitis, were included. The study protocol was approved by the Ethical Committee of the Faculty of Medicine, Gaziantep University (Ethical committee resolution no: 06-2009/237, date: 18.06.2009), and followed the tenets of the Helsinki Declaration. The study was funded by the Commission of Scientific Research Projects, Gaziantep University (Project no: TF.12.31).

Definitive diagnoses of meningitis were made following clinical and radiological evaluation by the Department of Infectious Diseases, on the basis of CSF biochemical indicators and observation of cells directly under the 
microscope. Although 22 patients diagnosed with bacterial or viral meningitis were initially included, one patient was lost during follow-up. Patients diagnosed with tuberculous meningitis were excluded.

During the first study visit, written informed consent was obtained from the patients or their relatives, as required. After collecting detailed medical histories and conducting examinations, patients who fell into any of the following groups were excluded:

- A history of any intracranial pathologies (brain tumor, cerebral abscess, etc.) and/or surgical operations

- $\quad$ Previous mild, moderate, or severe head trauma

- Hyperprolactinemia, hyperthyroidism, hypothyroidism or any other endocrinologic disorder that could lead to another anterior pituitary hormone disorder

- Meningitis, major depression, cerebrovascular disease that had been diagnosed within the last 6 months

- Had undergone hormone replacement therapy for any reason.

The height, weight, body mass index and waist circumference of all patients were measured at the time of initial admission and at 6 months, in order to evaluate the complications of any potential pituitary hormone deficiency. Routinely monitored serum sodium, potassium, blood urea nitrogen, calcium, phosphorus, fasting blood sugar, total protein, albumin, high-density lipoprotein, low-density lipoprotein, triglyceride, and total cholesterol levels were recorded. Polyuria symptoms were investigated to determine whether diabetes insipidus was present
At 24 hours after admission and at month 6, blood samples were collected from the antecubital vein in order to measure thyroid-stimulating hormone (TSH), growth hormone $(\mathrm{GH})$, follicle-stimulating hormone (FSH), luteinizing hormone (LH), prolactin (PRL), and adrenocorticotropic hormone (ACTH) levels. These blood samples were then centrifuged for 10 minutes at $4000 \mathrm{rpm}$ to separate out the serum. The serum samples were then placed into separate Eppendorf tubes labeled for TSH, GH, FSH, LH, PRL hormone, and ACTH, respectively, and stored at $-80^{\circ} \mathrm{C}$ until the study date. Measurements were made at the laboratory of the Department of Biochemistry, Faculty of Medicine, Gaziantep University Hospital. GH and ACTH levels were measured using immunoradiometric assay (Immulite-2000, DPC Cirrus, Inc., USA), while PRL. FSH, LH, and TSH levels were measured using radioimmunoassay (Architech C2000, Germany).

The distribution of the permanent data (the TSH, GH, FSH, LH, PRL, and ACTH levels) that were obtained in this study was examined graphically, using a KolmogorovSmirnov test. For the data that were normally distributed and dependent, groups were compared using a paired sample t-test, while for the data that were not normally distributed and dependent, groups were compared using a Wilcoxon test. The results were expressed in mean \pm standard deviation. We used SPSS for Windows, Ver. 18.0 (SPSS Inc., Chicago, Illinois, USA) for statistical evaluation. In all comparisons, $\mathrm{p}<0.05$ was considered statistically significant.

\section{Results}

Table 1: Patient characteristics, infectious disease, neurological deficits during acute CNS infection, and actual symptoms.

\begin{tabular}{|c|c|c|c|c|c|}
\hline $\begin{array}{l}\text { Patient } \\
\text { No }\end{array}$ & Age & Gander & Infectious diseasae & Neurological deficits & Actual symptoms \\
\hline 1 & 64 & M & Menengitis, unknown etiology & Paraparesis (legs) & Convulsion \\
\hline 2 & 32 & $\mathrm{~F}$ & Menengitis, unknown etiology & No & Cephalgia, fever \\
\hline 3 & 60 & M & Menengitis, unknown etiology & No & Fever, vomitting, confusion \\
\hline 4 & 44 & $\mathrm{~F}$ & Menengitis, unknown etiology & No & Cephalgia \\
\hline 5 & 29 & $\mathrm{~F}$ & Menengitis, unknown etiology & No & $\begin{array}{l}\text { Cephalgia, blurred vision, } \\
\text { double vision }\end{array}$ \\
\hline 6 & 25 & $\mathrm{~F}$ & Menengitis, unknown etiology & No & $\begin{array}{l}\text { Cephalgia, fever, vomitting } \\
\text { blurred vision, double vision }\end{array}$ \\
\hline 7 & 62 & M & Menengitis, unknown etiology & No & Vomitting, sickness, rinorea \\
\hline 8 & 68 & M & Herpes simplex menengitis & No & Fever \\
\hline 9 & 98 & $\mathrm{~F}$ & Menengitis, unknown etiology & No & Confusion \\
\hline 10 & 25 & $\mathrm{~F}$ & Menengitis, unknown etiology & No & $\begin{array}{l}\text { Cephalgia, fever, dizziness, } \\
\text { blurred vision, double vision }\end{array}$ \\
\hline 11 & 47 & $\mathrm{~F}$ & Menengitis, unknown etiology & No & Cephalgia \\
\hline 12 & 43 & M & Viral menengitis & No & Fever \\
\hline 13 & 42 & $\mathrm{~F}$ & Neuroborreliosis & No & Cephalgia \\
\hline 14 & 34 & M & Menengitis, unknown etiology & No & Cephalgia, fever \\
\hline 15 & 72 & $\mathrm{~F}$ & Menengitis, unknown etiology & No & Cephalgia \\
\hline 16 & 47 & $\mathrm{~F}$ & Menengitis, unknown etiology & No & Cephalgia \\
\hline 17 & 17 & M & Menengitis, unknown etiology & No & Fever, vomitting \\
\hline 18 & 27 & $\mathrm{~F}$ & Menengitis, unknown etiology & No & Fever \\
\hline 19 & 17 & $\mathrm{M}$ & Menengitis, unknown etiology & No & Fever \\
\hline 20 & 18 & M & Menengitis, unknown etiology & No & Fever, cephalgia \\
\hline 21 & 73 & $\mathrm{M}$ & Menengitis, unknown etiology & No & Cephalgia, fever \\
\hline
\end{tabular}


A total of $47.6 \%$ of participants were male, while $52.4 \%$ were female, with a mean age of 44,04 $\pm 21,73$ years (age range, 17-98) (Table 1). The admission FSH levels of two postmenopausal females were lower than normal, and these levels remained consistently low at month 6 . Although the admission FSH levels of five non-postmenopausal female patients were lower than normal, two of these women had normal levels at month 6, while one had higher than normal levels. The admission FSH level was higher than normal in one of the male patients, and these levels remained consistently high at month 6 . While the admission FSH levels for two of the male patients were normal, levels were below normal in one patient and above normal in the other at month 6 (Table 2).

Table 2: Hormone concentrations at the acute phase and at 6 months after acute infectious meningitis.

\begin{tabular}{|c|c|c|c|c|c|c|c|}
\hline $\begin{array}{l}\text { Hormones } \\
\text { Patients }\end{array}$ & Gander & TSH & АСТН & GH & FSH & LH & PROLAKTIN \\
\hline \multirow{2}{*}{1} & \multirow{2}{*}{ M } & $0.7^{*}$ & 21 & 0.27 & 42.75 & 15.51 & 11.73 \\
\hline & & $1.42 \alpha$ & 17 & 0.19 & 3.18 & 4.66 & 15 \\
\hline \multirow{2}{*}{2} & \multirow{2}{*}{ F } & 0.31 & 24.8 & 7.87 & 1.29 & 2.04 & 22.82 \\
\hline & & 1.53 & 6.24 & 7.75 & 1.33 & 1.93 & 22 \\
\hline \multirow{2}{*}{3} & \multirow{2}{*}{ M } & 0.78 & 18.5 & 0.05 & 1.53 & 0.29 & 71.24 \\
\hline & & 1.79 & 24.6 & 0.05 & 2.34 & 1.21 & 12 \\
\hline 4 & $\mathrm{~F}$ & 0.92 & 4.98 & 0.05 & 3.02 & 1.17 & 5 \\
\hline \multirow{2}{*}{5} & \multirow{2}{*}{$\mathrm{F}$} & 1.48 & 21.3 & 2.49 & 15.37 & 10.81 & 17.32 \\
\hline & & 2.54 & 26.9 & 0.05 & 14.92 & 9.87 & 16 \\
\hline \multirow{2}{*}{6} & \multirow{2}{*}{$\mathrm{F}$} & 1.99 & 55.8 & 0.05 & 3.74 & 4.29 & 11.11 \\
\hline & & 1.8 & 71.1 & 0.05 & 4.75 & 6.41 & 11 \\
\hline \multirow{2}{*}{7} & \multirow{2}{*}{ M } & 1.21 & 17.4 & 2.39 & 4.06 & 3.97 & 7.22 \\
\hline & & 2.55 & 7.52 & 0.05 & 3.96 & 2.78 & 6.8 \\
\hline 8 & M & 1.61 & 10.8 & 2.19 & 12.35 & 7.49 & 11 \\
\hline \multirow{2}{*}{9} & \multirow{2}{*}{$\mathrm{F}$} & 1.36 & 24.7 & 1.65 & 1.23 & 0.2 & 15.13 \\
\hline & & 1.27 & 18.6 & 1.17 & 19.53 & 8.06 & 31 \\
\hline \multirow{2}{*}{10} & \multirow{2}{*}{$\mathrm{F}$} & 1.24 & 14.8 & 0.09 & 6.24 & 5.78 & 10.09 \\
\hline & & 1.24 & 6.38 & 1.54 & 3.31 & 5.63 & 15 \\
\hline \multirow{2}{*}{11} & \multirow{2}{*}{$\mathrm{F}$} & 0 & 32.5 & 0.23 & 1.64 & 0.27 & 33.94 \\
\hline & & 2.13 & 42.6 & 0.05 & 2.03 & 0.9 & 30 \\
\hline \multirow{2}{*}{12} & \multirow{2}{*}{ M } & 0.54 & 27.6 & 2.92 & 1.12 & 0.94 & 18.66 \\
\hline & & 0.58 & 25.8 & 2.73 & 1.16 & 0.88 & 17.86 \\
\hline 13 & $F$ & 0.82 & 29.5 & 0.05 & 2.24 & 2.91 & 20.28 \\
\hline 13 & $\mathrm{~F}$ & 0.69 & 21 & 0.1 & 2.36 & 2.34 & 8.6 \\
\hline & & 0.69 & 25.6 & 0.18 & 6 & 4.38 & 59.18 \\
\hline 14 & $\mathrm{M}$ & 0.47 & 18 & 0.25 & 6.06 & 4.26 & 12 \\
\hline 15 & & 1.15 & 24.9 & 2.12 & 5.04 & 3.97 & 8.96 \\
\hline 15 & $\mathrm{~F}$ & 1.24 & 21.8 & 0.05 & 4.96 & 4.03 & 7.1 \\
\hline 16 & $F$ & 1.11 & 13.5 & 1.21 & 4.44 & 6.42 & 412.98 \\
\hline 17 & M & 1.68 & 40.6 & 1.19 & 5.32 & 9.58 & 19 \\
\hline 18 & $\mathrm{~F}$ & 2.64 & 7.44 & 6.03 & 10.62 & 2.5 & 14.8 \\
\hline 18 & $\mathrm{~F}$ & 2.11 & 57.9 & 0.76 & 2.01 & 1.45 & 40 \\
\hline & & 0.59 & 5 & 1.66 & 1.84 & 1.62 & 13.12 \\
\hline 19 & $\mathrm{M}$ & 0.9 & 32.9 & 0.05 & 2.53 & 2.04 & 12 \\
\hline 20 & $M$ & 0.18 & 5 & 0.26 & 0.05 & 0.75 & 8.06 \\
\hline 20 & M & 0.18 & 3.9 & 0.68 & 0.05 & 0.64 & 43 \\
\hline 21 & $M$ & 0.79 & 61.5 & 0.18 & 5.45 & 1.35 & 30.85 \\
\hline 21 & VI & 0.75 & 57.5 & 0.17 & 5.38 & 1.33 & 27 \\
\hline
\end{tabular}

The LH levels of two postmenopausal women were lower than normal on admission, and evaluation at month 6 showed that these levels remained consistently low. The LH levels of three non-postmenopausal women were lower than normal on admission, and while evaluation at month 6 showed normal levels in two of the women, levels remained consistently low in the third woman. Conversely, although LH levels were normal in two of the nonpostmenopausal women on admission, they were lower than normal at month 6. Furthermore, the low LH level that was observed in one of the male patients on admission remained consistently low at month 6 (Table 2).

The TSH levels of four patients were lower than normal on admission. In three of these individuals, levels were normal at month 6 , but remained consistently low in the fourth patient. PRL levels were higher than normal in five patients on admission. At month 6 , three of these patients had normal levels, while levels remained consistently high in the other two. Conversely, although the PRL levels of three patients were normal on admission, levels were high 
in all three at month 6 . At month 6 , six patients who had had normal GH levels on admission now had lower than normal levels. The GH levels of four patients were lower than normal on admission, while at month 6 , levels were normal in two of these patients, while remaining consistently low in the other two (Table 2).

Four patients with normal ACTH levels on admission had low levels at month 6. Two patients with higher than normal ACTH levels on admission also had high levels at month 6. Conversely, three patients had lower than normal ACTH levels on admission. Of these individuals, one had normal values at month 6 , while one still had low levels, and the other had higher than normal levels.

TSH $(p=0,008)$, LH $(p=0,662)$, and ACTH $(p=0,961)$ levels were higher at month 6 than on admission. Although the difference in TSH level was statistically meaningful, the increases in $\mathrm{LH}$ and ACTH were insignificant (Table). Conversely, GH ( $\mathrm{p}=0,094)$, FSH $(\mathrm{p}=0,334)$, and PRL ( $\mathrm{p}=$ 0,218 ) levels were lower at month 6 than they were on admission, although this decrease was not statistically significant (Table 3).

Table 3. Hormone concentrations at the acute phase and at 6 months after acute infectious meningitis

\begin{tabular}{llll}
\hline Hormones & Bazale $(\mathbf{n : 2 1})$ & $\mathbf{6}$ months $(\mathbf{n : 2 1})$ & $\mathbf{p *}, \mathbf{a}$ \\
\hline TSH & $0.97 \pm 0.68$ & $1.47 \pm 0.70$ & $0.008^{*}$ \\
GH & $1.44 \pm 2.07$ & $0.82 \pm 1.74$ & $0.094 \mathrm{a}$ \\
FSH & $6.12 \pm 9.33$ & $5.2 \pm 4.8$ & $0.334 \mathrm{a}$ \\
LH & $3.7 \pm 3.8$ & $4.07 \pm 2.97$ & $0.662^{*}$ \\
PRL & $41.14 \pm 89.22$ & $36.71 \pm 83.40$ & $0.218 \mathrm{a}$ \\
& & & $0.961^{*}$ \\
ACTH & $24.57 \pm 15.14$ & $24.7 \pm 19.88$ & \\
\hline
\end{tabular}

Thyroid-stimulating hormone (TSH), growth hormone (GH), folliclestimulating hormone (FSH), luteinizing hormone (LH), prolactin (PRL), and adrenocorticotropic hormone $(\mathrm{ACTH}) . \mathrm{P} *$ : Paired T test, $\mathrm{P}^{\mathrm{a}}$ : Wilcoxon test.

\section{Discussion}

Prospective-retrospective studies and isolated case reports have shown an incidence of increased pituitary insufficiency following CNS infections [1,4-10]. However, these studies are not sufficient in number [5-7]. The present study prospectively investigated this issue, and found that hormonal instability occurs in patients with infectious meningitis.

It has been evident for some time that tuberculous meningitis causes pituitary insufficiency [3]. In 1978, Hagg et al. reported non-tuberculous bacterial or viral meningitis and pituitary insufficiency for the first time, in two cases [11]. It was found that coxsackie B5 was the underlying factor in one of these cases, while gonadotropic, thyrotrophic, and somatotropic insufficiency occurred in both. Kupari et al. (1980) isolated the Herpes simplex virus in one of three patients with viral meningitis, and the influenza-A virus in another [8]. Somatotropic and corticotropic insufficiency occurred in two of these cases, while panhypopituitarism, and diabetes insipidus developed in one of them. Lichtenstein et al. (1982) reported that panhypopituitarism, hyperprolactinemia, and diabetes insipidus developed in one patient with viral meningitis with an unknown underlying factor [9], and Lew et al. (1984), reported the development of corticotropic insufficiency and diabetes insipidus in a patient with bacterial meningitis with an unknown underlying factor [12]. Ickenstein et al. (1999) described the development of corticotropic, thyrotrophic and gonadotropic insufficiency, hyperprolactinemia, and diabetes insipidus in a patient with meningitis associated with Herpes simplex, and Vesely et al. (2000) found that the latter was also associated with the development of thyrotrophic and gonadotropic insufficiency in patients with meningitis $[10,13]$. Katsuno et al. (2003) reported a low level of pituitary hormones in a female patient aged 27 who had been diagnosed with viral meningitis [14], while Lynch et al. (2007) found that hypopituitarism developed in one patient who had been diagnosed with bacterial meningitis [15].

To date, only five systematic studies (three retrospective and two prospective) have assessed pituitary dysfunction due to acute infectious meningitis [1,4-7]. In the first retrospective study, Schaefer et al. [1] evaluated 19 patients with a history of viral or bacterial meningitis/encephalitis, at a mean duration of 26 months after the acute event. Of these patients, four (21\%) had isolated corticotropic deficiency and two (11\%) showed borderline gonadotropic insufficiency. None had somatotropic or thyrotrophic insufficiency or evidence of diabetes insipidus. In the second retrospective study, the pituitary functions of 14 patients with acute bacterial or viral meningitis were investigated [4]. The mean evaluation time was 20 months after acute meningitis, and four patients (28.6\%) had isolated $\mathrm{GH}$ deficiency. The remaining pituitary axes were considered normal on the basis of basal hormone levels. In a very recent study, Levy-Shraga et al. evaluated the pituitary functions of 14 children with a history of meningitis [7], and reported that, in contrast to findings in adult patients, pituitary dysfunction with overt clinical symptoms was not a frequent consequence of acute meningitis.

In the first prospective study, Tsiakalos et al. demonstrated the risk of hypopituitarism during the acute phase and at 12 months after onset of acute viral and bacterial meningitis [5]. Of the 16 patients studied, 31.2\% showed pituitary hormone deficiencies, two showed gonadotropic deficiency, and three showed somatotropic deficiency in the acute phase, on the basis of basal hormone levels. At 12 months, five of the patients had at least one pituitary hormone deficiency (two had isolated ACTH 
deficiency, one had isolated GH deficiency, and two had combined GH and ACTH deficiency), on the basis of basal hormone levels and an insulin tolerance test.

In the second prospective study, Tanriverdi et al. [6] evaluated GH, ACTH, FSH, and LH levels during the acute phase of meningitis, and at 6 and at 12 months in 16 patients. They found that $18.7 \%$ of patients had $\mathrm{GH}$ deficiency, and $12.5 \%$ had ACTH and FSH/LH deficiencies in the acute phase. Furthermore, at 12 months, six of 16 patients $(42.8 \%)$ had GH deficiency, four had isolated $\mathrm{GH}$ deficiency, one had combined GH and ACTH deficiency, and one had combined GH and FSH/LH deficiency.

In our study of the activity of anterior pituitary hormones in 21 individuals with meningitis, mean TSH, LH, and ACTH levels were higher at month 6 than on admission. This difference was significant for TSH ( $\mathrm{p}=0.008)$, but insignificant for LH $(\mathrm{p}=0.662)$, and ACTH (0.961). Mean GH, FSH, and PRL levels were lower at month 6 than on admission. However, these decreases were not statistically meaningful. In case-based evaluations; FSH was low in seven patients, LH was low in six patients, TSH was low in four patients, $\mathrm{GH}$ was low in four patients and ACTH was low in three patients on admission, while FSH was low in five patients, LH was low in four patients, TSH was low in one patient, GH was low in six patients, and ACTH was low in five patients at month 6 (Table 2).

The mechanisms of pituitary dysfunction after acute meningitis are not clearly defined, but the pituitary hormone deficiency pattern is suggestive of a dynamic ongoing process. It is reasonable to assume that the incidence and pattern of hormonal deficiencies after acute infectious meningo-encephalitis may vary with the type of causative agent, and the localization of brain lesion, as well as with the severity of disease. Hormonal deficiencies may be transient or permanent. Tanriverdi et al. [6] showed the occurrence of anti-pituitary antibodies (APA) and antihypothalamus antibodies (AHA) for the first time, during the acute phase of meningitis, as well as 6 and 12 months later. The frequency of antibody positivity ranged from 35 to $50 \%$ of the patients throughout the 12 -month period. The presence of a substantially high frequency of AHA and APA after acute meningitis suggests that an autoimmune hypothalamic-pituitary process could be triggered by acute meningitis. However, an analysis of the relationship between pituitary dysfunction and the presence of the antibodies at any time during the follow-up period showed no significant correlation. The lack of correlation was assumed to be due to the short duration of the prospective follow-up.

Our study may have several limitations. First, the number of patients was low; we believe the results would be of greater significance if a higher number of patients had been studied. Another important limitation is the fact that we did not use stimulation tests to evaluate pituitary function.

In conclusion, on the basis of the present study, and in a similar manner to previous results obtained in adult patients, it may be that pituitary dysfunction develops following an infectious disease of the CNS. Furthermore, prospective studies are required to clarify the possible mechanisms underlying pituitary dysfunction during and following such infectious diseases.

\section{Conflict of Interest}

No conflict of interest was declared by the authors.

\section{References}

[1] Schaefer S, Hypothalamic-pituitary insufficiency following infectious diseases of the central nervous system. Eur J Endocrinol 2008;158:3-9.

[2] Schneider HJ, Kreitschmann-Andermahr I, Ghigo E, Stalla GK, Agha A Hypothalamopituitary dysfunction following traumatic brain injury and aneurysmal subarachnoid hemorrhage: a systematic review. JAMA 2007;298:1429-38.

[3] Haslam RHA, Winternitz WW, Howieson J Selective hypopituitarism following tuberculous menigitis. Am J Dis Child 1969;118:903-8.

[4] Tanriverdi F, Alp E, Demiraslan H, Dokmetas HS, Unluhizarci K, Doganay M, Casanueva FF, Kelestimur F Investigation of pituitary functions in patients with acute meningitis: a pilot study. J Endocrinol Invest 2008;31:489 491.

[5] Tsiakalos A, Xynos ID, Sipsas NV, Kaltsas G Pituitary insufficiency after infectious meningitis: a prospective study. J Clin Endocrinol Metab 2010;95:3277-3281 .

[6] Tanriverdi F, De Bellis A, Teksahin H, Alp E, Bizzarro A, Sinisi AA, et al. Prospective investigation of pituitary functions in patients with acute infectious meningitis: is acute meningitis induced pituitary dysfunction associated with autoimmunity? Pituitary 2012;5:579-588.

[7] Levy-Shraga Y, Gazit I, Modan-Moses D, Pinhas-Hamiel O. Pituitary function in children following infectious diseases of the central nervous system. Pituitary. 2013 Mar 8. DOI 10.1007/s11102-013-0476-2.[Epub ahead of print].

[8] Kupari M, Pelkonen R, Valtonen V. Post-encephalitic hypothalamic-pititary insufficiency. Acta Endocrinol 1980; 94: 433-438.

[9] Lichtenstein MJ, Tilley WS, Sandler MP. The syndrome of hypothalamic hypopituitarism complicating viral meningoencephalitis. J Endocrinol Invest 1982; 5: 111-115.

[10] Ickenstein GW, Klotz JM, Langohr HD. Virus encephalitis with symptomatic Parkinson syndrome, diabetes insipidus and panhypopituitarism. Fortschr Neurol Psychiatr 1999;67:476-481.

[11] Hagg E, Astrom L, Steen L. Persistent hypothalamicpituitary insufficiency following acute meningoencephalitis. Acta Medica Scandinavica 1978;203:231-235.

[12] Jew K, Piziak V, Gilliland PF \& Hurley DL. Meningoencephalitis complicated by pituitary insufficiency and a spontaneously resolving suprasellar mass. Neurosurgery $198414567-569$. 
[13] Vesely DL, Mastrandrea P, Samson C, Argyelan G \& Charvit S. Post-herpes encephalitic anterior pituitary insufficiency with hypothermia and hypotension. American Journal of the Medical Sciences 2000;320:273-277.

[14] Katsuno M, Yamazaki M, Tahara S, Murai Y, Teramono A, Sano N. Spontaneous remission of acromegaly after meningitis: a case report. No To Shinkei 2003;55:967-971.
[15] Lynch C, Fish M. Bacterial meningitis resulting in visual impairment and panhypopituitarism. $\mathrm{Br} \mathrm{J}$ Hosp Med 2007;68:272. 